\title{
Structure-Dependent Hematological Effects of Per- and Polyfluoroalkyl Substances on Activation of Plasma Kallikrein-Kinin System Cascade
}

Qian S. Liu, ${ }^{\dagger, \ddagger}$ Yuzhen Sun, ${ }^{\dagger, \S}$ Guangbo Qu, ${ }^{\dagger, \ddagger \odot ~ Y a n m i n ~ L o n g, ~}{ }^{\S}$ Xingchen Zhao, ${ }^{\dagger \neq}$ Aiqian Zhang, ${ }^{\dagger, \ddagger(0)}$

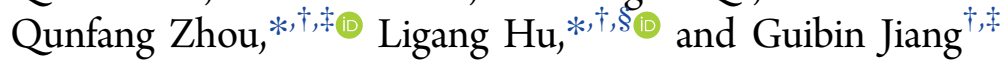

${ }^{\dagger}$ State Key Laboratory of Environmental Chemistry and Ecotoxicology, Research Center for Eco-Environmental Sciences, Chinese Academy of Sciences, Beijing, 100085, P. R. China

${ }^{\ddagger}$ College of Resources and Environment, University of Chinese Academy of Sciences, Beijing, 100049, P. R. China

${ }^{\S}$ Institute of Environment and Health, Jianghan University, Wuhan, 430000, P. R. China

Supporting Information

ABSTRACT: Per- and polyfluoroalkyl substances (PFASs) are a global concern because of their ubiquitous occurrence and high persistence in human blood, and increasing amounts of unidentified fluorinated compounds are now becoming new exposure issues. This study aims to investigate the structurerelated effects of PFASs on the activation of the plasma kallikreinkinin system (KKS). The effects of 20 PFASs and the related longchain aliphatic compounds were screened, and their binding affinities for the initial zymogen, Hagmen factor XII (FXII) in the KKS, were evaluated by molecular docking analysis. PFASs were demonstrated to activate the KKS in a structure-dependent mode.

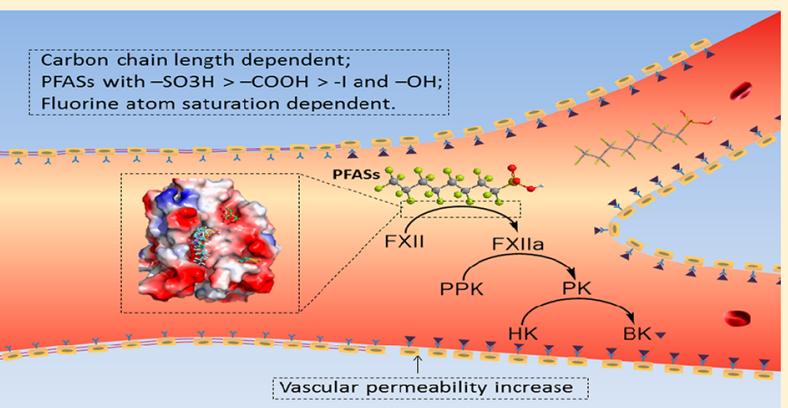
More specifically, PFASs with longer carbon chain length, higher fluorine atom substitution degree, and terminal acid group exhibited relatively higher activities in activating the KKS. The binding affinities of PFASs with FXII determined their capabilities for inducing KKS activation. The alternative binding modes of PFASs with FXII, together with van der Waals and hydrogen bonds, specifically accommodated the distinctive chemical structures. To our knowledge, PFASs, for the first time, were found to induce the activation of the KKS in plasma, and their chemical structure-related effects would be extremely important for risk assessment on emerging PFASs in addition to the listing in Stockholm Convention.

\section{INTRODUCTION}

Per- and polyfluoroalkyl substances (PFASs) are a large group of anthropogenic chemicals that have been extensively used in industrial and commercial products worldwide. ${ }^{1,2}$ The unintended release of PFASs during the production, usage, and disposal of the related products poses widespread exposure risks for wildlife and human beings. As the most prevalent members in PFASs, both perfluorooctanesulfonic acid (PFOS) and perfluorooctanoic acid (PFOA) have drawn global attention because of their ubiquitous occurrence in human blood samples. ${ }^{3,4}$ Exceptionally high serum PFOS concentrations were found in fluorochemical production workers (up to $36625 \mathrm{ng} / \mathrm{mL}$ ) and fishery employees $(10400 \mathrm{ng} / \mathrm{mL}$, median level) ${ }^{5,6}$ Considering their high persistence in blood ${ }^{4}$ and the substantial toxicological data, ${ }^{7-9}$ PFOS, PFOA, and their salts, precursors, and related products have been included or are proposed to be included in the listing under the Stockholm Convention. ${ }^{10,11}$

The commitment toward national as well as international regulations contributed to the declining trends in blood PFOS and PFOA concentrations in the Unites States, Sweden, and
Germany. ${ }^{12-14}$ Nevertheless, the body burden of the prevalent PFASs remains high in some developing countries due to occupational exposure and lax management. ${ }^{5,6,15}$ Moreover, the levels of some other PFASs, such as the long-chain perfluorinated carboxylic acids (PFCAs), were reported to elevate in human blood during 1980 to $2010 .^{13,16-18}$ The temporal study showed an increasing amount of unidentified organofluorine in blood samples, suggesting that people are facing new exposure risks from the application of emerging PFAS alternatives. ${ }^{19}$ Accordingly, the numerous potential PFAS congeners and alternatives, whose toxicological properties could be comparable to the prevalent members due to high structure similarity, are of great concern but still represent a poorly understood family of environmental contaminants. Understanding the structure-related toxicological effects is

Received: April 20, 2017

Revised: July 22, 2017

Accepted: July 26, 2017

Published: July 26, 2017 
thus important for the emerging PFASs with regarding to their potential health risks.

Diverse toxicities, including hepatotoxicity, reproductive and developmental toxicity, endocrine disrupting effect, immunotoxicity, and neurotoxicity, have been reported for PFASs, especially for PFOS and PFOA, ${ }^{7,8,11,20,21}$ while the data on potential hematological effects of PFASs remain sparse. Given the widespread occurrence and persistence of PFASs in human blood, the question of whether they stay inert or actively react with plasma proteins and exert adverse effects would be interesting to study with respect to explaining their hazards to human health.

As one of the key components in plasma, the kallikrein-kinin system (KKS), consisting of Hageman factor XII (FXII), plasma prekallikrein (PPK), and high-molecular-weight kininogen $(\mathrm{HK})$, plays important roles in regulating multiple biological responses such as inflammation, blood pressure, coagulation, and vascular permeability, etc. ${ }^{22-24}$ Some clinical symptoms such as hereditary angioedema, which is sometimes life-threatening, are attributed to the loss in the homeostasis of KKS activation. ${ }^{25}$ The activation of PPK via a FXII-dependent mechanism induces vascular dysfunction and retinal thickening in diabetic rats, ${ }^{26}$ mediates brain hemorrhage and edema in mice after stroke. ${ }^{27}$ Besides the endogenous factors, the KKS is vulnerable to the exogenous stimulators, and its activation has been employed to evaluate the potential hematological effect and the resulting vascular permeability of emerging chemicals such as silver nanoparticles. ${ }^{24}$ As the autoactivation of FXII zymogen initiates KKS activation, the FXII activators are crucial in the regulation of this cascade. ${ }^{28}$ PFASs have high structural similarities to natural long-chain fatty acids, an internal FXII activator, $^{29}$ and thus could possibly trigger the KKS cascade as well. The screening of PFASs as the potential KKS activators is thus important in understanding their toxicological effects in blood and the underlying molecular mechanisms.

In this study, we evaluated a series of PFASs with distinct structural characteristics for their potential hematological effects based on KKS activation. The PPK cleavage was induced by PFASs in a structure-dependent manner. The waterfall cascade activation of the KKS was subsequently characterized. The binding affinity of PFASs with FXII determined their capabilities in activating the KKS. This study discussed the structure-dependent hematological effects of PFASs based on KKS activation, which provided new indications for their potential health risks from the ubiquitous occurrence of these kinds of emerging chemicals in human blood.

\section{MATERIALS AND METHODS}

2.1. Ethical Approval. All the experiments involving animals were conducted in accordance with International Guidelines. Animal experiments were supervised by the Animal Care and Use Committee of Research Center for EcoEnvironmental Sciences, Chinese Academy of Sciences. The research involving human subjects were approved by the Institutional Review Board (IRB) of Research Center for EcoEnvironmental Sciences, Chinese Academy of Sciences and complied with all relevant national, state, and local regulations. Informed consent was obtained from all individual participants included in the study.

2.2. Reagents. Six chemicals, including perfluorohexanesulfonate (PFHxS, 98\%), perfluorooctanoic acid (PFOA, 96\%), perfluorodecanoic acid (PFDA, 98\%), perfluorododecanoic acid (PFDoA, 95\%), perfluorotetradecanoic acid (PFTA, 96\%), and octanoic acid (98\%), were purchased from Sigma-Aldrich (U.S.). Two of the PFASs, perfluorohexadecanoic acid (PFHxDA, 95\%) and perfluorooctadecanoic acid (PFOcDA, 97\%), were bought from Alfa Aesar (U.S.). The other 12 chemicals tested in this study, which included PFOS (98\%), perfluorohexanoic acid (PFHxA, 97\%), perfluorohexyl iodide (PFHxI, 98\%), perfluorooctyl iodide (PFOI, 98\%), 4:2 FTOH (97\%), 5:1 FTOH (98\%), 6:2 FTOH (98\%), 7:1 FTOH (98\%), 1-octanesulfonic acid sodium salt (99.5\%), 6:2 fluorotelomer sulfonate (6:2 FTS, 98\%), 5:3 acid, and palmitic acid (97.5\%), are commercially available from J\&K Scientific (U.S.). The chemical structures of these compounds are depicted in SI Figure S1. The stock solutions (10-50 mM) were made by dissolving the corresponding chemicals in dimethyl sulfoxide (DMSO, Sigma-Aldrich) and kept at $4{ }^{\circ} \mathrm{C}$ in darkness until use. The working solutions were prepared by diluting the stock solutions with sterile saline. The vehicle of DMSO $(<2 \%)$, which showed no significant difference from the blank one (data not shown), was used as the negative control in all experiments. All the other reagents used were of analytical grade or higher, unless otherwise stated.

2.3. Ex Vivo PPK Activation Assay. The system for ex vivo PPK activation assay consisted of $9 \mu \mathrm{L}$ of freshly collected mouse plasma (C57BL/6, Vital River Laboratory Animal Technology Co. Ltd.) and $1 \mu \mathrm{L}$ of stimulation solution containing PFASs, and the reaction was performed at $37{ }^{\circ} \mathrm{C}$. Kaolin $(1 \mathrm{mg} / \mathrm{mL})$ was tested as the positive control. Three representative compounds, including PFOA, PFOS, and PFHxDA, were first selected for the dose-response and time course analyses of ex vivo PPK activation. For the doseresponse, a series of concentrations of PFOA $(0,1,3,5 \mathrm{mM})$, PFOS $(0,1,3,5 \mathrm{mM})$, and PFHxDA $(0,1,10,30,50,100,500$ $\mu \mathrm{M})$ were tested, with an incubation time of $2 \mathrm{~h}$. With respect to the time course, time points of $0,5,15,30,60$, and $120 \mathrm{~min}$ were monitored when the particular concentrations of PFOA (5 mM), PFOS $(5 \mathrm{mM})$, and PFHxDA $(100 \mu \mathrm{M})$ were controlled.

The stimulation was terminated by the direct addition of the sample loading buffer (Laemmli buffer, containing $4 \%$ bmercaptoethanol) and processed for subsequent Western blot assay. Briefly, equal amounts of plasma samples (i.e., $0.5 \mu \mathrm{L}$ of plasma in $10 \mu \mathrm{L}$ of sample buffer) from different treatments were loaded onto mini SDS-PAGE (4-20\% gradient, Bio-Rad) for protein separation. The subsequent membrane transferring (nitrocellulose, Pall), immunoblotting (PPK primary antibody from $R \& D, 1: 1000$, peroxidase-conjugated rabbit antigoat second antibodies from ZSGB-BIO, 1:500) and development (Pierce ECL, Thermo Scientific) were performed for the analysis of PPK and PK expression levels in plasma samples. The pixel densities on the developed X-ray film were analyzed using Quantity One software, and the extent of PPK activation was expressed as the ratio of PK to PPK.

2.4. Tests for Structure-Related Effect of PFASs on PPK Activation. For determining the influence of structural factors in PFASs, the compounds containing different carbon chain lengths, terminal groups, and fluorine atom substitution degrees were classified and examined using the same incubation system for ex vivo PPK activation assay described above. For carbon chain length, three groups of PFASs containing 6-8 carbon atoms $(\mathrm{C} 6-8)$ terminated with $-\mathrm{SO}_{3} \mathrm{H}(\mathrm{PFHxS}$, PFOS, $5 \mathrm{mM}), 6-10$ carbon atoms $(\mathrm{C} 6-10)$ terminated with $-\mathrm{COOH}$ (PFHxA, PFOA, PFDA, $5 \mathrm{mM}$ ), and 10-18 carbon atoms (C10-18) terminated with $-\mathrm{COOH}$ (PFDA, 


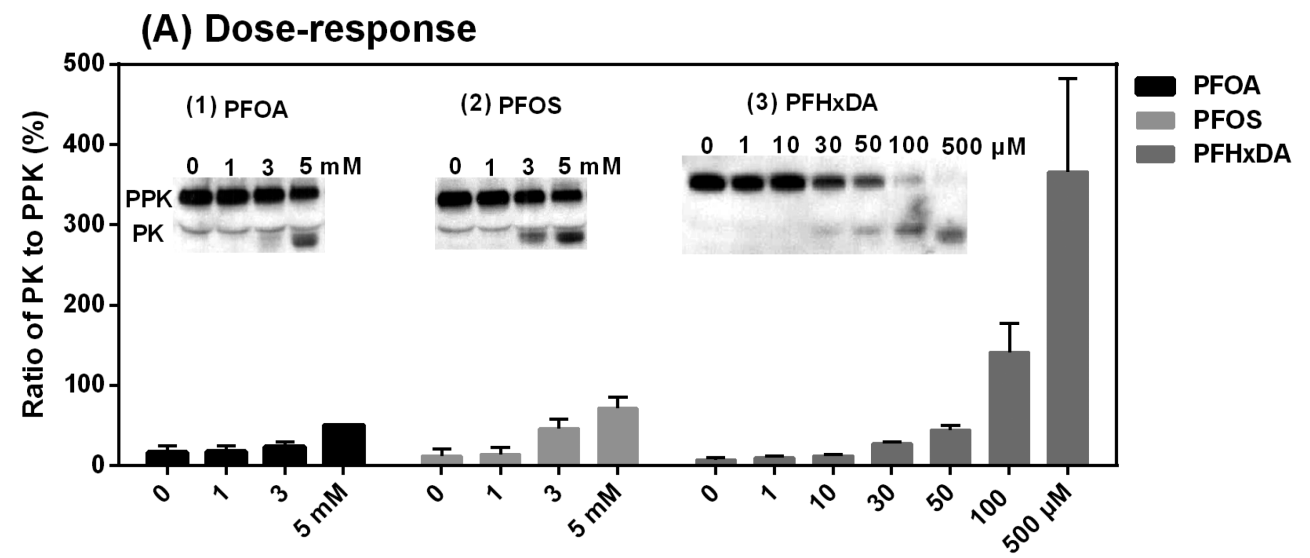

(B) Time course

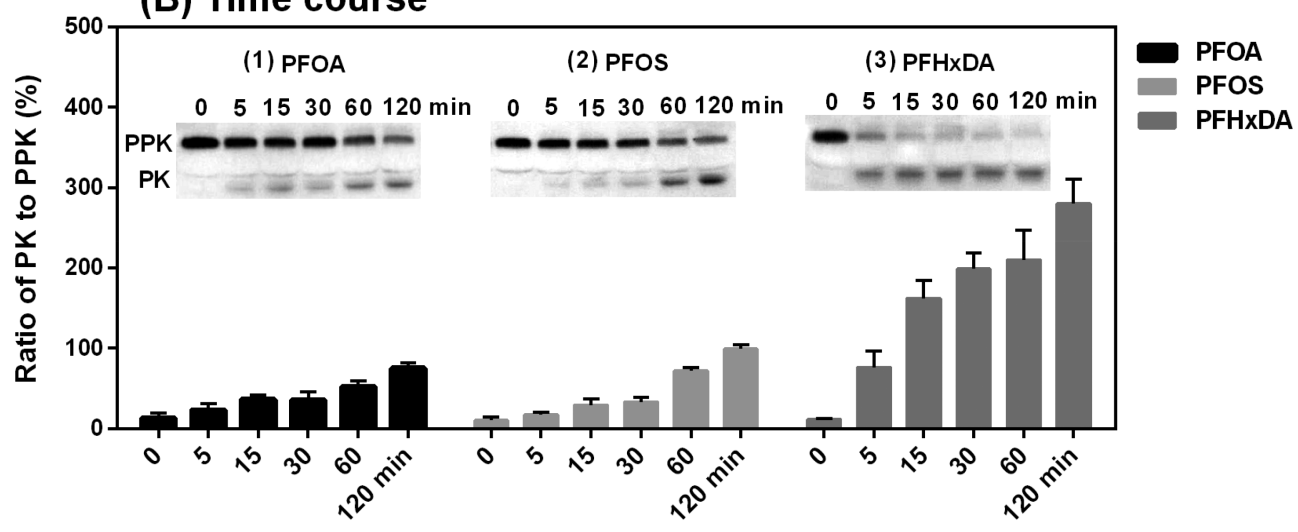

(C) Enzymatic activities
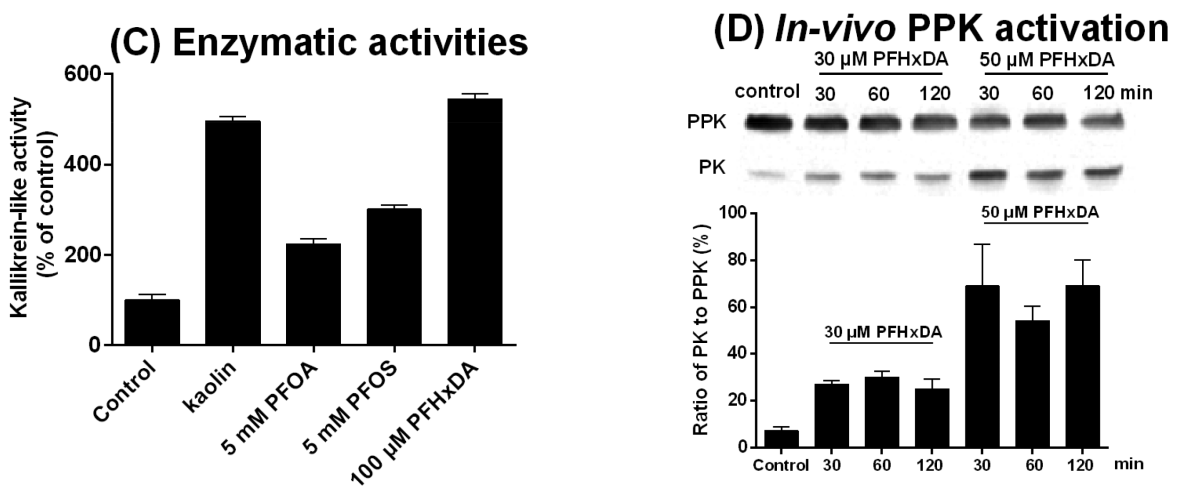

Figure 1. PPK cleavage activation was induced by PFAS exposure. (A) Dose-dependent PPK activation ex vivo. The incubation time was $2 \mathrm{~h}$. (1) PFOA, (2) PFOS, (3) PFHxDA. (B) Time course for PFAS-induced PPK activation ex vivo. (1) $5 \mathrm{mM}$ PFOA, (2) $5 \mathrm{mM}$ PFOS, (3) $100 \mu \mathrm{M}$ PFHxDA. (C) PFAS-exposure increased kallikrein-like activities in mouse plasma. (D) Tail vein injection of PFHxDA induced PPK activation in vivo.

PFDoA, PFTA, PFHxDA, PFOcDA, $100 \mu \mathrm{M}$ ) were compared. For the terminal group, two groups of PFASs containing C6 (PFHxI, 4:2 FTOH, 5:1 FTOH, PFHxA, PFHxS, $5 \mathrm{mM}$ ) and C8 (PFOI, 6:2 FTOH, 7:1 FTOH, PFOA, PFOS, $3 \mathrm{mM}$ ) terminated with $-\mathrm{I},-\mathrm{OH},-\mathrm{COOH}$, and $-\mathrm{SO}_{3} \mathrm{H}$, respectively, were evaluated. In view of the fluorine atom substitution degree, three groups of PFASs and aliphatic compounds containing $\mathrm{C} 8$ terminated with $-\mathrm{SO}_{3} \mathrm{H}$ (1-octanesulfonic acid sodium salt, 6:2 FTS, PFOS, $5 \mathrm{mM}$ ), C8 terminated with $\mathrm{COOH}$ (octanoic acid, 5:3 acid, PFOA, $5 \mathrm{mM}$ ), and $\mathrm{C} 16$ terminated with $-\mathrm{COOH}$ (palmitic acid, PFHxDA, $100 \mu \mathrm{M}$ ) were analyzed. The stimulation time was $2 \mathrm{~h}$, and the samples were subsequently processed for Western blot.

2.5. In Vivo PPK Activation Assay. Eight-week-old male C57BL/6 mice with an average weight of $22 \mathrm{~g}$ were purchased from Vital River Laboratory Animal Technology Co. Ltd.
(Beijing, China). All the materials used on the mice were sterile, and the experiments were performed under sterile condition. PFHxDA was selected to test in vivo PPK activation, and it was delivered to $\mathrm{C} 57 \mathrm{BL} / 6$ mice by tail vein injection at the dose of $4 \mu \mathrm{L} / \mathrm{g}$ body weight. The final stimulation concentrations were controlled at $30 \mu \mathrm{M}$ or $50 \mu \mathrm{M}$ in blood, and the circulation lasted for 30,60, and $120 \mathrm{~min}$, respectively. DMSO $(0.5 \% \mathrm{v} / \mathrm{v}, 120 \mathrm{~min})$ was used as the vehicle control. When the exposure was terminated, all the animals were sacrificed by $\mathrm{CO}_{2}$ inhalation, and the mouse blood was immediately collected to prepare plasma samples for subsequent PPK Western blot as described previously. All in vivo treatments were performed on three independent mice.

2.6. Kallikrein-Like Activity Assay. The mouse plasma samples were preactivated by $1 \mathrm{mg} / \mathrm{mL}$ Kaolin, $5 \mathrm{mM}$ PFOA, 5 $\mathrm{mM}$ PFOS, or $100 \mu \mathrm{M}$ PFHxDA for $15 \mathrm{~min}$, respectively. After 
centrifugation, $4 \mu \mathrm{L}$ of the supernatant plasma samples were added in a Corning 96-well black plate, wherein $95 \mu \mathrm{L}$ of 10 mM PBS ( $\mathrm{pH} 7.4$ ) with $50 \mu \mathrm{M} \mathrm{ZnSO}_{4}$ was preloaded in each well. The monitoring of the fluorescence $(\lambda \mathrm{ex} / \mathrm{em}: 400 \mathrm{~nm} /$ $505 \mathrm{~nm}$ ) was immediately started after adding $1 \mu \mathrm{L}$ of $2 \mathrm{mM}$ fluorogenic kallikrein substrate (H-D-Val-Leu-Arg-AFC-2HCl, Calbiochem, EMD Millipore) and continued for $30 \mathrm{~min}$ at an interval of $30 \mathrm{~s}$ using the multifunction microplate reader (Thermo Scientific, VARIOSKAN FLASH).

2.7. KKS Cascade Activation Assay. The in vitro KKS activation assay was performed using human purified zymogens of FXII, PPK, and HK. The serine protease PK $(0.94 \mu \mathrm{M})$ was used as the positive control. All purified proteins were purchased from Enzyme Research Laboratories. In $25 \mu \mathrm{L}$ of $10 \mathrm{mM}$ PBS buffer system containing $50 \mu \mathrm{M} \mathrm{ZnSO}_{4}(\mathrm{pH} 7.4)$, $5 \mathrm{mM}$ PFOS or $100 \mu \mathrm{M}$ PFHxDA was incubated with $0.58 \mu \mathrm{M}$ FXII, a mixture of $0.58 \mu \mathrm{M}$ FXII and $0.94 \mu \mathrm{M}$ PPK, or a mixture of $0.58 \mu \mathrm{M}$ FXII, $0.94 \mu \mathrm{M}$ PPK, and $0.36 \mu \mathrm{M}$ HK at 37 ${ }^{\circ} \mathrm{C}$ for $2 \mathrm{~h}$. Kaolin $(1 \mathrm{mg} / \mathrm{mL})$ was tested as the positive control. All the samples were subsequently prepared for Western blot assays. The primary antibodies of FXII (1:1000, Cedarlane Laboratories), PPK (1:1000, Abcam), and HK (1:2000, R\&D), coupled with the corresponding secondary antibodies (1:500, ZSGB-BIO), were used for immunoblotting. For studying the effect of HSA, 5 and $10 \mathrm{~g} / \mathrm{L}$ HSA were premixed with FXII $(0.58 \mu \mathrm{M})$ and subsequently stimulated by PFHxDA $(100 \mu \mathrm{M})$ and PFOS $(5 \mathrm{mM})$ for $2 \mathrm{~h}$, respectively. The samples were subsequently processed for FXII activation analysis by Western blot as described above.

For ex vivo KKS cascade activation, human plasma (healthy volunteers from the lab) was activated by $1 \mathrm{mg} / \mathrm{mL}$ Kaolin, 5 $\mathrm{mM}$ PFOS, or $100 \mu \mathrm{M}$ PFHxDA using a similar reaction system in ex vivo PPK activation assay. The plasma samples were processed for subsequent Western blot of FXII and HK.

2.8. Native PAGE Analysis. The binding between the chemicals and FXII zymogen was evaluated by native PAGE gels. Briefly, kaolin $(1 \mathrm{mg} / \mathrm{mL}$, positive control), PFOS $(0,1,3$ $\mathrm{mM})$, and PFHxDA $(0,1,10,50,100 \mu \mathrm{M})$ were incubated with $0.58 \mu \mathrm{M}$ FXII purified protein in $25 \mu \mathrm{L}$ of $10 \mathrm{mM}$ PBS with 50 $\mu \mathrm{M} \mathrm{ZnSO}_{4}$ ( $\left.\mathrm{pH} 7.4\right)$ for $2 \mathrm{~h}$, respectively. The unbound FXII from each treatment was separated on $8 \%$ native PAGE gel, transferred, and immunoblotted by FXII primary antibody (1:1000, Cedarlane Laboratories) on nitrocellulose membranes. The amount of free FXII reversely represented the binding degree of FXII with the tested activators.

2.9. Spectroscopic aAnalysis. The fluorescence spectra of FXII protein $(0.58 \mu \mathrm{M})$ was monitored using a spectrofluorimeter (Horiba, Fluoromax-4, Edison NJ) with $\lambda$ ex of 280 $\mathrm{nm}$ and $\lambda \mathrm{em}$ in the range of $300-450 \mathrm{~nm}$. The changes in fluorescent profiles were evaluated for FXII upon $2 \mathrm{~h}$ treatment of $1 \mathrm{mg} / \mathrm{mL}$ kaolin, $500 \mu \mathrm{M}$ PFOS, or $100 \mu \mathrm{M}$ PFHxDA, which indicated the interactions between the protein and the tested activators.

The UV absorbance at $280 \mathrm{~nm}$ was also measured for 0.58 $\mu \mathrm{M}$ FXII after treatment with $5 \mathrm{mM}$ PFOS or $100 \mu \mathrm{M}$ PFHxDA by using a spectrophotometer (DR5000, HACH).

2.10. Molecular Docking Analysis. The idock program ${ }^{30}$ was applied to search the interaction pattern between FXII and the 20 tested compounds in this study. Similar to the docking method of AutoDockVina, ${ }^{31}$ idock adopts an optimization algorithm to explore the conformational space and predicts the binding affinity of ligand-protein. The protease domain of crystal structure of human FXII was extracted from the protein database (PDB id: 4XDE) as the receptor structure of docking. The cocrystal ligand and water molecules were removed before the docking simulation. The geometric structures of the tested compounds were optimized at B3LYP/6-311++G(d,p) level by Gaussian 09 software (Revision B.01, Gaussian, Inc.: Wallingford, CT, 2009). The necessary input files of receptors and ligands were produced using the AutodockTools v1.5.6. ${ }^{32}$ During the docking process, the receptor structure containing the entire protease domain was kept rigid and the ligands were treated as the flexible structures. For each compound, 500 docking runs were performed, and the conformation with the most negative docking score was then selected for subsequent analysis of the binding mode.

2.11. Statistical Analysis. At least triplicate parallel experiments were performed independently for each assay, and all results were expressed as mean value \pm SD.

\section{RESULTS}

3.1. PFAS-Caused PPK Activation. As indicated previously, ${ }^{24} \mathrm{KKS}$ activation was characterized by PPK cleavage concomitant with plasma kallikrein (PK) formation in mouse plasma. The feasibility of ex vivo assay for PPK activation in mouse plasma was confirmed by the test of the positive control Kaolin (see SI, Figure S2A). Three representative PFASs, including PFOA, PFOS, and PFHxDA, were selected to test their potential in activating the KKS. As indicated in Figure 1A, all these PFASs caused dose-dependent PPK activation after $2 \mathrm{~h}$ treatment, indicating these kinds of emerging chemicals are potential new KKS activators. According to the activation extent of PPK characterized by Western blot, the approximate lowest-observed-effect concentrations (LOECs) were $5 \mathrm{mM}$ for PFOA, $3 \mathrm{mM}$ for PFOS, and $30 \mu \mathrm{M}$ for PFHxDA. This implied that PFASs with different chemical structures had distinct capabilities in activating the KKS.

The studies on the time courses for these three chemicals (Figure 1B) showed that ex vivo PPK activation occurred immediately after the addition of the chemicals in the plasma (within $5 \mathrm{~min}$ ), and the activation extent obviously increased with the prolonged incubation time $(0-120 \mathrm{~min})$. PFHxDA caused the most efficient effect when compared with the other two tested chemicals, which was consistent with the results in Figure 1A.

To further confirm the capabilities of PFASs in activating the KKS, ex vivo enzymatic assay based on the fluorogenic kallikrein substrate was characterized (see SI, Figure S2B) and performed. Figure $1 \mathrm{C}$ shows that similar to the positive control Kaolin, the addition of these three PFASs clearly elevated the kallikrein-like activities in mouse plasma, confirming their roles in the mediation of PPK activation. In agreement with the findings from Figure $1 \mathrm{~A}$ and $\mathrm{B}$, the PFASinduced kallikrein-like activities followed the order of PFOA < PFOS < PFHxDA; the stimulation concentration of PFHxDA $(100 \mu \mathrm{M})$ was 50 folds lower than those of the other two compounds ( $5 \mathrm{mM})$.

The effects of PFASs on KKS activation were also tested in vivo using a mouse model. Figure $1 \mathrm{D}$ shows that tail vein injection of PFHxDA caused obvious PPK cleavage in mouse plasma. According to the ratios of $\mathrm{PK} / \mathrm{PPK}$ relative to the vehicle control, PFHxDA-induced PPK activation occurred in a dose-dependent manner, and this effect was sustained for the test duration $(120 \mathrm{~min})$. Therefore, PFASs in the blood circulation system could efficiently cause KKS activation in vivo. Nevertheless, in contrast to ex vivo assay, in vivo PPK 
(A) Carbon chain length

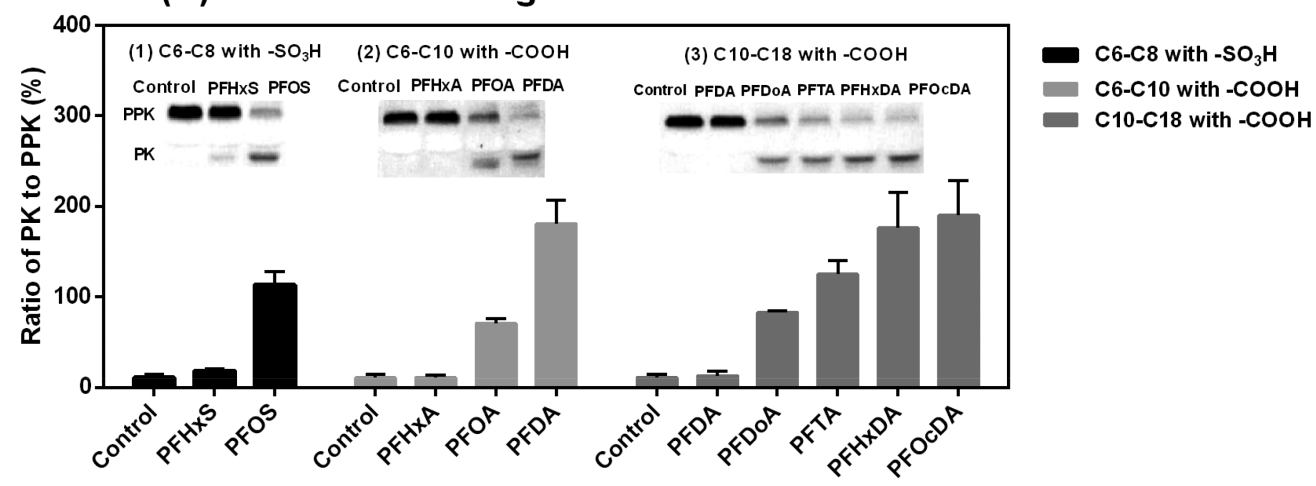

(B) Terminal group

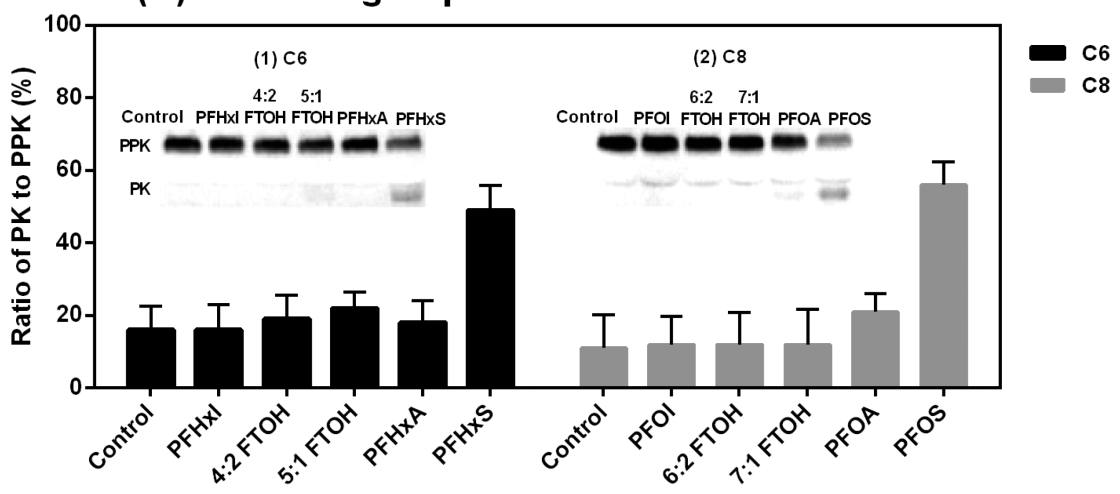

(C) Fluorine atom substitution degree

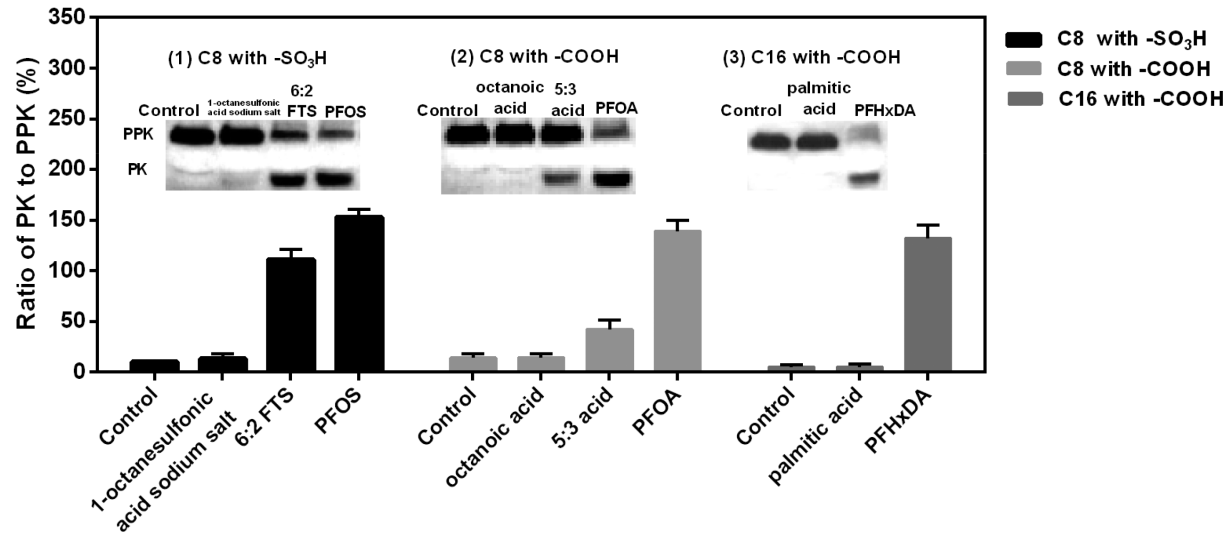

Figure 2. Structure-dependent effects of PFASs on PPK activation. The incubation time was $2 \mathrm{~h}$. (A) The influence of carbon chain length on PFASinduced PPK activation. (1) C6-C8 PFASs terminated with $-\mathrm{SO}_{3} \mathrm{H}(5 \mathrm{mM})$ : control, PFHxS, PFOS; (2) C6-C10 PFASs terminated with $-\mathrm{COOH}(5 \mathrm{mM})$ : control, PFHxA, PFOA, PFDA; (3) C10-C18 PFASs terminated with -COOH (100 $\mu \mathrm{M})$ : control, PFDA, PFDoA, PFTA, PFHxDA, PFOcDA. (B) The influence of terminal groups on fluorinated aliphatic compound-induced PPK activation. (1) C6 fluorinated aliphatic compounds (5 mM): control, PFHxI, 4:2 FTOH, 5:1 FTOH, PFHxA, PFHxS; (2) C8 fluorinated aliphatic compounds (3 mM): control, PFOI, 6:2 FTOH, 7:1 FTOH, PFOA, PFOS. (C) The influence of fluorine atom substitution degree on fluorinated aliphatic compound-induced PPK activation. (1) $\mathrm{C} 8$ aliphatic compounds terminated with $-\mathrm{SO}_{3} \mathrm{H}(5 \mathrm{mM})$ : control, 1-octanesulfonic acid sodium salt, 6:2 FTS, PFOS; (2) C8 aliphatic compounds terminated with $-\mathrm{COOH}(5 \mathrm{mM})$ : control, octanoic acid, 5:3 acid, PFOA; (3) C16 aliphatic compounds terminated with $-\mathrm{COOH}(100 \mu \mathrm{M})$ : control, palmitic acid, PFHxDA.

activation did not exhibit in a typical time-dependent manner, which indicated the presence of intricate KKS regulatory mechanisms in mice. ${ }^{24}$

3.2. Structure-Related Effect of PFASs on PPK Activation. As indicated above, PFASs can cause the PPK cleavage, and the activation extents can be different due to the chemical structure diversity. To evaluate their structure-related effects on KKS activation, a total of 20 PFAS congeners and alternatives with different carbon chain lengths, terminal groups, and fluorine atom substitution degrees (see SI, Figure S1) were selected. For the structural factor of carbon chain length, three groups of compounds terminated with either $-\mathrm{SO}_{3} \mathrm{H}$ or $-\mathrm{COOH}$ were compared at the optimized concentrations $(5 \mathrm{mM}$ or $100 \mu \mathrm{M})$. The results shown in Figure $2 \mathrm{~A}$ revealed that the PPK activation followed the order PFOS $>$ PFHxS (Figure 2A-1) and PFOcDA $>$ PFHxDA $>$ PFTA $>$ PFDoA $>$ PFDA $>$ PFOA $>$ PFHxA (Figure 2A-2, 2A- 
(A) In-vitro KKS activation

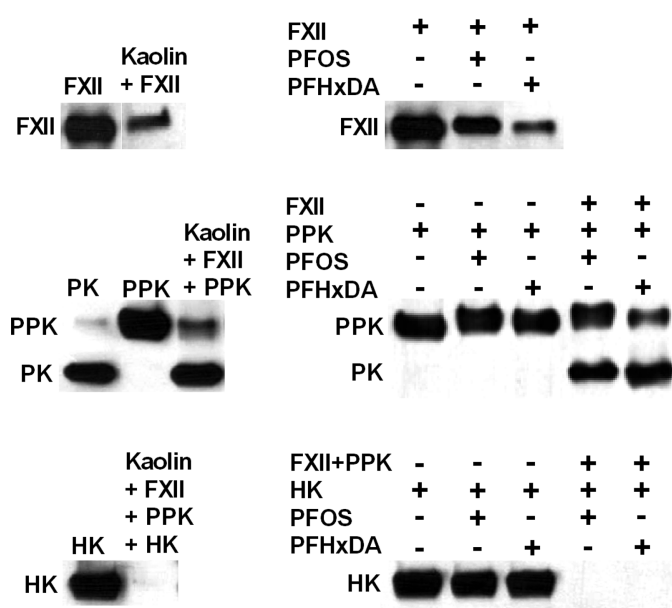

(B) Ex-vivo KKS activation

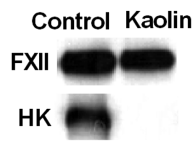

Control PFOS

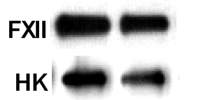

Control PFHxDA

FXII

HK

Figure 3. PFASs caused the waterfall cascade activation of the KKS both in vitro and ex vivo. (A) In vitro KKS cascade activation using purified human proteins. The reaction time was $2 \mathrm{~h}$. The concentrations of FXII, PK, PPK, and HK were 0.58, 0.94, 0.94, and 0.36 $\mu \mathrm{M}$, respectively. The tested activators included $1 \mathrm{mg} / \mathrm{mL}$ Kaolin (positive control, left panel), $5 \mathrm{mM}$ PFOS, and $100 \mu \mathrm{M}$ PFHxDA (right panel). (B) Ex vivo KKS cascade activation in human plasma. The stimulation lasted for $2 \mathrm{~h}$. The stimulators were $1 \mathrm{mg} / \mathrm{mL}$ Kaolin (positive control, upper panel), $5 \mathrm{mM}$ PFOS (middle panel), and $100 \mu \mathrm{M}$ PFHxDA (bottom panel).

A

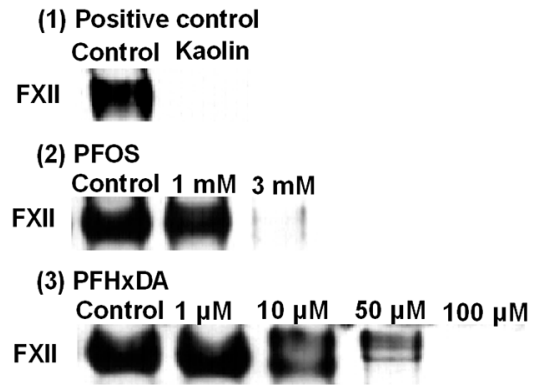

C

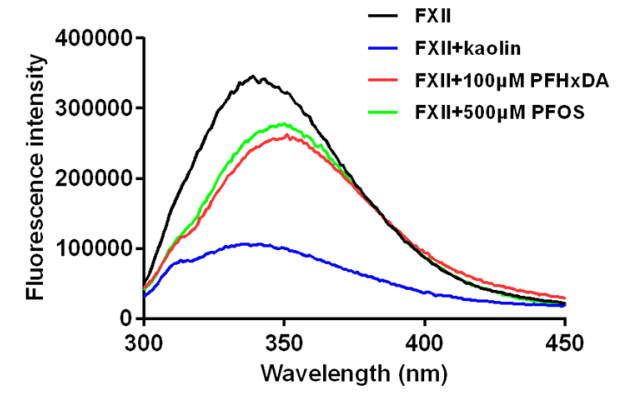

B

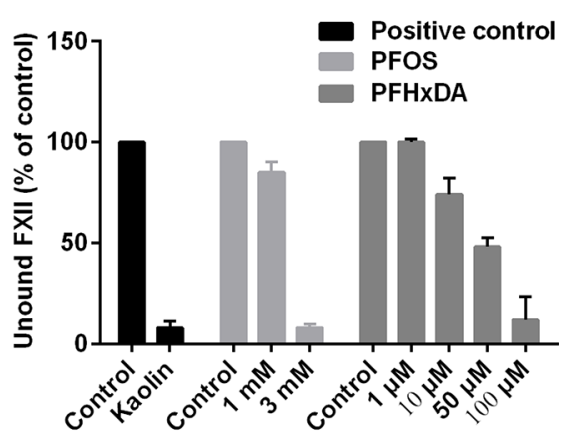

D

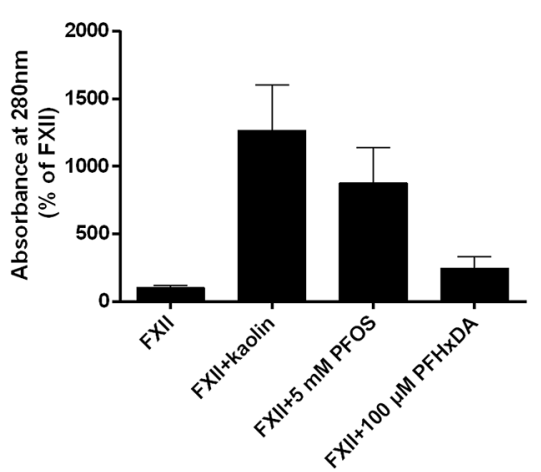

Figure 4. The binding of PFASs with FXII. (A) Native PAGE analysis of unbound FXII upon $2 \mathrm{~h}$ treatments of diverse activators, including (1) positive control (1 mg/mL kaolin), (2) PFOS (1, $3 \mathrm{mM}$ ), and (3) PFHxDA (1, 10, 50, and $100 \mu \mathrm{M})$. (B) Quantitative analysis for unbound FXII tested by native PAGE analysis. The alterations in intrinsic fluorescence (C) and absorbance at $280 \mathrm{~nm}$ (D) of $0.58 \mu \mathrm{M}$ FXII with the incubations of $1 \mathrm{mg} / \mathrm{mL}$ Kaolin, $500 \mu \mathrm{M}$ PFOS, and $100 \mu \mathrm{M}$ PFHxDA, respectively, for $2 \mathrm{~h}$.

3). It is obvious that the longer the carbon chain length of the chemical, the higher is its capability of activating the KKS.

The terminal groups of the fluorinated aliphatic compounds tested in this study included iodo $(-\mathrm{I})$, hydroxyl $(-\mathrm{OH})$, carboxyl $(-\mathrm{COOH})$, and sulfonic acid groups $\left(-\mathrm{SO}_{3} \mathrm{H}\right)$. Both 6-carbon (C6 series) and 8-carbon (C8 series) aliphatic compounds with diverse terminal groups were investigated. The results of the C6 series in Figure 2B-1 shows that only
PFHxS caused obvious PPK activation, while the others showed no effects. When the C8 series was compared (Figure 2B-2), both PFOA and PFOS exhibited detectable PPK activation, while the effects of PFASs with iodo or hydroxyl terminal groups were negligible. The PPK activation extent followed the order PFHxS > PFHxA and PFOS > PFOA (Figure 2B); thus, PFASs terminated with $-\mathrm{SO}_{3} \mathrm{H}$ were believed to have relatively higher capabilities of inducing KKS activation than those 
A-1

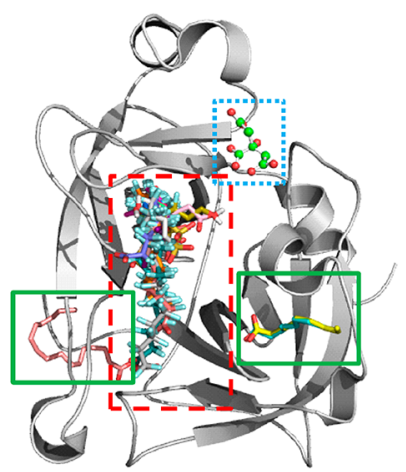

B-1

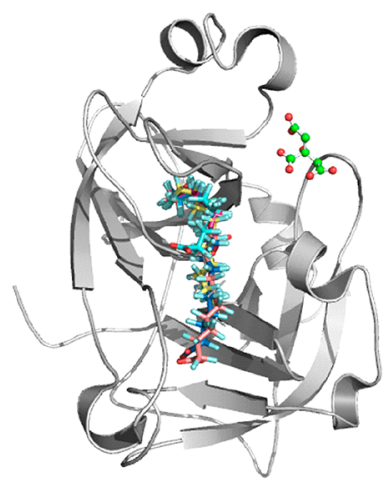

A-2

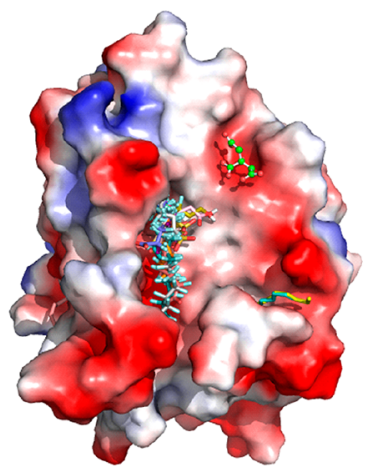

B-2

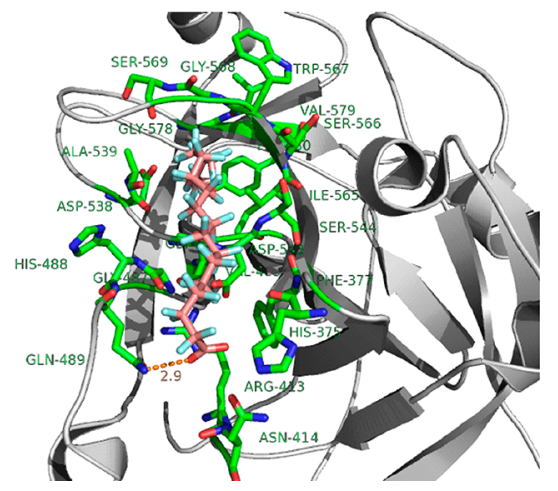

Figure 5. Predicted binding modes of FXII with PFASs and the related long-chain aliphatic compounds. (A-1) Predicted binding sites of FXII with PFASs and the related aliphatic compounds. Blue frame: original FXII ligand; left green frame: palmitic acid; right green frame: octanoic acid; red frame: all the other compounds. (A-2) Vacuum electrostatic surface of the catalytic domain of FXII (residues 359-599). Molecular representations were generated using the PyMOL 1.1 software for qualitative evaluation. The potential surface was colored in a red (negative)-blue (positive) spectrum in the $\pm 64 \mathrm{kT} / \mathrm{e}$ range. (B-1) Overlay of binding sites for PFASs with different carbon chain lengths. (B-2) Depiction of the binding mode between FXII and PFOcDA.

terminated with $-\mathrm{COOH}$ or the other terminal groups, such as $-\mathrm{OH}$ and $-\mathrm{I}$.

With respect to fluorine atom substitution degree, we tested three groups of compounds, that is, $\mathrm{C} 8$ terminated with $-\mathrm{SO}_{3} \mathrm{H}$, C8 terminated with $-\mathrm{COOH}$, and $\mathrm{C} 16$ terminated with $-\mathrm{COOH}$. PPK activation was observed in the following orders: PFOS $>6: 2$ FTS $>1$-octanesulfonic acid sodium salt (Figure 2C-1), PFOA > 5:3 acid > octanoic acid (Figure 2C-2), and PFHxDA > palmitic acid (Figure 2C-3). These findings indicate that the higher the fluorine atom substitution degree of the PFAS, the greater is its effect on KKS activation. Altogether, PFAS-induced KKS activation is closely related to their chemical structures, including the carbon chain length, terminal groups, and the fluorine atom substitution degree.

3.3. PFAS-Triggered Waterfall Cascade Activation of the KKS. The KKS cascade consists of three main zymogens, i.e., FXII, PPK, and HK. ${ }^{22}$ To investigate how PFASs work on the KKS, the in vitro assays based on the purified human plasma zymogens were performed. As indicated by the stimulation of the positive control Kaolin, the waterfall activation of FXII, PPK, and HK was distinctly characterized by the weakening or elimination of the corresponding zymogen bands and the elevated expression of PK band (Figure 3A, left panel). When the representative compounds of PFOS (the most concerned form of PFASs) and PFHxDA (the most efficient chemical in activating the KKS in this study) were tested, the results in Figure 3A (right panel) showed they both directly caused the obvious activation of FXII. In contrast to FXII, neither PPK nor HK could be directly influenced by the addition of PFASs. Alternatively, these two zymogens were activated by the coaddition of upstream zymogen(s) of FXII or FXII plus PPK with the chemicals of PFOS or PFHxDA. This finding confirmed that PFASs triggered the waterfall cascade activation of the KKS, and this process was initiated through FXII autoactivation upon the chemicals.

When compared to the in vitro reaction system based on purified human protein or the ex vivo system of mouse plasma, human plasma is complicated and can have distinct biological regulatory mechanisms. The potential effects of PFASs on ex vivo $\mathrm{KKS}$ activation was also investigated in human plasma, and the results are shown in Figure 2B. Similar to Kaolin (positive control, upper panel), the incubation of PFOS (middle panel) or PFHxDA (bottom panel) evidently reduced or diminished both FXII and HK zymogen bands in human plasma, proving that the KKS waterfall cascade activation was induced therein as well.

3.4. the Binding of PFASs with FXII. As indicated in Figure 3, the autoactivation of FXII, the initial zymogen of the KKS, is necessary for the subsequent activation of PPK and HK zymogens (Konings et al., 2011). Therefore, the interaction between PFASs and FXII is very crucial for KKS cascade activation. To identify the binding of PFASs on FXII, native PAGE separation coupled with FXII Western blot assay was performed. In contrast to the free FXII, the migration of the binding complex (i.e., FXII-activators) would be restrained in native PAGE due to the conformational changes and increased size of the protein, ${ }^{24}$ leading to a reduction in the free FXII band visualized on native PAGE. We found that the positive control Kaolin efficiently bound with FXII to form the complex, leaving no free protein entering the gel (Figure 4A1). The $2 \mathrm{~h}$ 
incubation of FXII with PFOS $(1,3 \mathrm{mM})$ or PFHxDA $(1,10$, 50 , and $100 \mu \mathrm{M})$ distinctly reduced the amount of free FXII entering the gel, and the protein band reduction was observed in a dose-related manner (Figure 4A2, 4A3), which confirmed the occurrence of the binding of FXII with PFASs.

As FXII contains the fluorophoric aromatic amino acid Trp, it has intrinsic fluorescence (see SI, Figure S3) with the $\lambda$ ex/em 280/350 nm (Colman and Schmaier 1997; Ren et al. 2015). ${ }^{33,34}$ The binding of FXII with the activator Kaolin changed its intrinsic fluorescence intensity (Figure 4C), due to the conformational change of the protein and the microenvironmental hydrophilicity alteration of Trp. ${ }^{33,34}$ Similarly, the incubation of PFOS and PFHxDA also greatly reduced the intrinsic fluorescent intensities of FXII, which confirmed the binding of FXII with PFASs.

FXII shows an absorption peak at $280 \mathrm{~nm}$, attributed to $\mathrm{n}-\pi^{*}$ transition of the fluorophoric aromatic amino acid Trp. ${ }^{33,35}$ The UV absorbance changes are considered as another effective indicator for structural alterations due to FXII-chemical complex formation. As shown in Figure 4D, the incubation of Kaolin obviously enhanced the absorbance of FXII at $280 \mathrm{~nm}$. Similar to Kaolin, PFOS and PFHxDA clearly increased the absorbance of FXII at $280 \mathrm{~nm}$ as well, indicating the binding of PFASs with FXII, which was in line with the results from native PAGE assay and intrinsic fluorescence analysis (Figure 4A-C).

3.5. The Structure-Related Binding Affinities of PFASs with FXII. PFAS induced KKS activation in a structure-related manner (Figure 2) and the binding of PFAS with FXII was crucial for KKS activation (Figure 3 and 4). Thus, in silico molecular docking analysis was further used to characterize the influence of chemical structural characteristics on their binding affinities. SI Table S1 shows the docking scores for the binding of FXII with PFASs and the related long-chain aliphatic compounds as indicated in SI Figure S1. The lower the score is, the stronger is the binding affinity of the chemical with FXII. When the structural characteristics of the carbon-chain length, terminal group, and fluorine atom substitution degree were evaluated, the docking score orders were consistent with the ex vivo PPK activation extent (Figure 2), with the exception of PFHxI due to the special characteristics of iodine. The results suggested that the capabilities of PFASs in inducing KKS activation were dependent on their structure-related binding affinities with FXII zymogen. That is, the higher binding affinity of the chemical with FXII caused more severe KKS activation.

The binding modes between PFASs and FXII are depicted in Figure 5A. The blue frame indicated the cocrystal FXII ligand, the green ones implied octanoic acid or palmitic acid, and all the other compounds are boxed with the red frame. PFASs mainly bound to the surface groove in the center of FXII protein in contrast to the corresponding fatty acids. The binding modes of PFASs with different carbon chain lengths are overlaid in Figure 5B-1. Among these compounds, PFOcDA with the longest carbon chain exhibited the largest interaction area. More specifically, the hydrophobic residues in the binding pocket (Phe377, Val425, Gly487, Ala539, Trp567, Gly568, Gly578, and Val579) showed van der Waals interactions with the $\mathrm{CF}_{3}\left(\mathrm{CF}_{2}\right)_{16}$ - structure of PFOcDA, suggesting that they played an important role in stabilizing the fluorocarbon chain of the ligand. In addition, the nitrogen atom of amide side chain in Gln489 formed a hydrogen bond of $2.9 \AA$ with the carboxyl group of $\mathrm{PFOcDA}$, indicating that electrostatic interactions were also important to recognize the polar group of PFOcDA (Figure 5B-2). When compared with PFOcDA, the other tested chemicals bound to FXII with less van der Waals interactions due to the decreases in fluorinated carbon chain lengths. As for hydrogen bonds, distinctive compounds had different situations. Some compounds, including PFHxA, PFDoA, PFHxI, PFOI, and Octanoic acid did not form hydrogen bonds with FXII, whereas the other compounds potentially formed hydrogen bonds with different amino acid sites on FXII. For examples, chemicals including PFOA, PFHxS, 1-Octanesulfonic acid sodium salt, and 6:2FTS had hydrogen bond interactions with Ser544 of FXII. PFDA and PFOS potentially interacted with His488 of FXII. The molecular docking analysis showed the rest could have single or multiple hydrogen bond interaction sites on FXII (e.g., PFHxDA: Gln489; PFTA: Arg413; 5:3 acid: Ser544, His393; Palmitic acid: Arg362; 4:2 FTOH: Cys571; 5:1 FTOH: Cys571, Ser569; 6:2 FTOH: His393; 7:1 FTOH: His393, Ser566, and Ser544). The binding modes for all the tested chemicals were specifically depicted in SI Figure S4 and S5, implying that the shadow binding cavity of FXII surface could accommodate different carbon chain lengths of PFASs by the alternative binding modes. Therefore, on the basis of the binding modes described above, we could infer that the binding of PFASs on the surface pocket of FXII might influence the structural changes in the protein and induce consequent biological effects.

\section{DISCUSSION}

PFASs are ubiquitously present in blood and organs with abundant blood supply, and nearly all people have PFAS contamination in their blood, regardless of age. ${ }^{36,37}$ Given the inevitable occurrence of PFASs and increasing trend of unidentified organofluorine in blood, understanding their structure-related interactions with physiologically relevant plasma protease would be of great importance in explaining their potential hematological effects and health risks. This study found that PFASs could bind with FXII zymogen in a structuredependent manner, which subsequently triggered the waterfall cascade activation of the KKS, thus showing the potential to induce diverse hematological effects, such as increased vascular permeability. ${ }^{24}$ When compared to the more prevalent members of PFOA and PFOS, which are included in international regulations, some fully fluorinated alkyl congeners with relatively longer carbon chains exerted higher capabilities in inducing KKS activation, suggesting that the potential health risks from the increasing exposure to new fluorinated congeners are of high concern.

According to the lowest-observed-effect concentration (LOECs) of PFASs in this study, it was found that PFOS and PFOA did not cause obvious PPK cleavage until their concentrations reached to $3 \mathrm{mM}$ and $5 \mathrm{mM}$, respectively (Figure 1A). Considering that serum PFOS or PFOA was around several to dozens $\mathrm{ng} / \mathrm{mL}$ in general populations, ${ }^{3}$ and reached around $200 \mu \mathrm{M}$ in professional populations, ${ }^{5,6,38}$ the hematological effects observed herein might not be physiologically relevant for these two compounds themselves in real scenarios. Nevertheless, PFASs with longer carbon chain, such as PFHxDA, could trigger KKS activation at the low dose to 30 $\mu \mathrm{M}$ (Figure 1A), and potentially regulate paracellular permeability in endothelial cells. ${ }^{24}$ The increasing unidentified fluorinated compounds in blood samples, originating from unintended exposure to newly marketed but proprietary PFAS alternatives, could thus pose a potential threat to human health, considering their long-term universal exposure and high bioaccumulation potentials. 
As the most abundant protein in plasma, albumin was previously reported to bind with PFASs, ${ }^{39}$ which could influence their interaction with other plasma proteases such as FXII. The effect of human serum albumin (HSA) on PFASinduced FXII activation was thus investigated using in vitro assay. The results showed that HSA had no effect on PFHxDAcaused FXII activation (see SI, Figure S6A), while it blocked PFOS-induced FXII cleavage in a concentration-dependent manner (see SI, Figure S6B). This finding indicated that albumin might preferentially pose the shielding function on the interaction between FXII and PFASs with relatively short carbon chain. When considering the binding modes of HSA and PFASs, PFOA showed higher interaction affinity for HSA as compared to PFOS, ${ }^{5}$ the interaction affinity declined due to steric hindrances associated with longer and more rigid perfluoroalkyl chains, ${ }^{40}$ and the binding constants of perflorinated carboxylic acids (PFCAs) were lower than those of fatty acids with the same carbon chain length. ${ }^{41}$ These distinctive binding behaviors well explained the potential shielding function of albumin on PFAS-induced KKS activation in the plasma, as the opposite structure-related effects were observed for PFASs from ex vivo assays (Figure 2) and molecular docking analysis (see SI, Table S1). In spite of the shielding function of albumin on PFAS-induced FXII activation, results from ex vivo and in vivo assays (Figures 1, 2, and 3B) indicated that PFASs could more or less activate the KKS in real plasma system.

Considering the new worries about increasing trends of PFAS congeners and alternatives in plasma, increasing focus is on their potential hazardous effects and the chemical structurerelevance. Structure-dependent effects of PFASs have been observed on the binding of human peroxidome proliferatoractivated receptor and thyroid hormone receptor (TR), cytotoxicities based on human colon carcinoma, and development of zebrafish in early life stages, and both fluorocarbon chain and terminal functional groups were correlated with the biological effects of PFASs. ${ }^{42-44}$ For instance, TR binding potency of PFASs was dependent on the terminal group in order of $-\mathrm{SO}_{3} \mathrm{H}>-\mathrm{COOH}$ by comparing PFBA with PFBS (C4), PFHxA with PFHxS (C6), and PFOA with PFOS (C8), respectively. ${ }^{45}$ The transthyretin binding potencies were higher for PFASs (C4-C8) containing $-\mathrm{SO}_{3} \mathrm{H}$ than for those containing $-\mathrm{COOH}^{46}$ According to the results from ex vivo PPK cleavage and molecular docking analysis in this study, the structures of terminal group, carbon chain length, and fluorine substitution degree in PFASs apparently played important roles in the mediation of KKS activation due to the different binding affinities of the chemicals with FXII zymogen (Figure 2, see SI, Table S1). That is, PFASs with the terminal polar acid group, longer carbon backbone chain, and higher fluorine atom substitution exhibited relatively higher capabilities in activating the KKS when compared to the analogues with specific structure characteristics controlled. The characteristic waterfall cascade activation of the KKS triggered by PFASs in the plasma offered substantial evidence on the potential hematological effects of suspicious PFAS congeners, besides the listing in Stockholm Convention, and the findings are extremely important for advisory guidance on the sound management of commercial PFAS alternatives.

In this study, PFASs were demonstrated to induce structurerelated KKS activation in blood, and the structural characteristics, including the terminal group, carbon chain length, and fluorine atom substitution degree, were crucially involved. The binding affinities of the chemicals with FXII determined the capabilities of PFASs in activating the KKS cascade through the sequential cleavage of FXII, PPK, and HK zymogens. The findings proved the structure-dependent hematological effects caused by PFASs, thus providing substantial evidence for new concerns on the emerging PFAS congeners and alternatives, besides the listing in Stockholm Convention.

\section{ASSOCIATED CONTENT}

\section{Supporting Information}

The Supporting Information is available free of charge on the ACS Publications website at DOI: 10.1021/acs.est.7b02055.

Additional information as noted in the text (PDF)

\section{AUTHOR INFORMATION}

\section{Corresponding Authors}

*(Q.Z) E-mail: zhouqf@rcees.ac.cn .

*(L.H.) Phone/fax: 86 10-62849334; e-mail: lghu@rcees.ac.cn. ORCID

Guangbo Qu: 0000-0002-5220-7009

Aiqian Zhang: 0000-0001-5680-2529

Qunfang Zhou: 0000-0003-2521-100X

Ligang Hu: 0000-0002-6213-4720

Notes

The authors declare no competing financial interest.

\section{ACKNOWLEDGMENTS}

This work was supported by the National Basic Research Program of China [grant number 2015CB453102]; the Major International (Regional) Joint Project (grant number 21461142001); the National Natural Science Foundation of China [grant numbers 21321004, 21477153]; and the Chinese Academy of Science (grant numbers 14040302, QYZDJ-SSWDQC017).

\section{ABBREVIATIONS}

DMSO dimethyl sulfoxide

FTOHs fluorotelomer alcohols

FTS fluorotelomer sulfonate

FXII Hagmen factor XII

HK high-molecular-weight kininogen

HSA human serum albumin

KKS kallikrein-kinin system

LOECs lowest-observed-effect concentrations

PFASs per- and polyfluoroalkyl substances

PFCAs perfluorinated carboxylic acids

PFDA perfluorodecanoic acid

PFDoA perfluorododecanoc acid

PFHxA perfluorohexanoic acid

PFHxDA perfluorohexadecanoic acid

PFHxI perfluorohexyl iodide

PFHxS perfluorohexanesulfonic acid

PFOA perfluorooctanoic acid

PFOcDA perfluorooctadecanoic acid

PFOI perfluorooctyl iodide

PFOS perfluorooctanesulfonic acid

PFTA perfluorotetradecanoic acid

PK plasma kallikrein

PPK plasma prekallikrein 


\section{REFERENCES}

(1) Günter, S.; Werner, S.; Andrew, F.; Bruce, S.; Fred, B.; Herward, V.; Blaine, M., Fluorine Compounds, Organic. In Ullmann's Encyclopedia of Industrial Chemistry; Wiley-VCH Verlag GmbH \& Co. KGaA, 2000.

(2) Wang, J.; Zhang, Y.; Zhang, W.; Jin, Y.; Dai, J. Association of perfluorooctanoic acid with HDL cholesterol and circulating miR-26b and miR-199-3p in workers of a fluorochemical plant and nearby residents. Environ. Sci. Technol. 2012, 46 (17), 9274-81.

(3) Kannan, K.; Corsolini, S.; Falandysz, J.; Fillmann, G.; Kumar, K. S.; Loganathan, B. G.; Mohd, M. A.; Olivero, J.; Van Wouwe, N.; Yang, J. H.; Aldous, K. M. Perfluorooctanesulfonate and related fluorochemicals in human blood from several countries. Environ. Sci. Technol. 2004, 38 (17), 4489-4495.

(4) Olsen, G. W.; Burris, J. M.; Ehresman, D. J.; Froehlich, J. W.; Seacat, A. M.; Butenhoff, J. L.; Zobel, L. R. Half-life of serum elimination of perfluorooctanesulfonate, perfluorohexanesulfonate, and perfluorooctanoate in retired fluorochemical production workers. Environ. Health Perspect. 2007, 115 (9), 1298-305.

(5) Gao, Y.; Fu, J.; Cao, H.; Wang, Y.; Zhang, A.; Liang, Y.; Wang, T.; Zhao, C.; Jiang, G. Differential accumulation and elimination behavior of perfluoroalkyl Acid isomers in occupational workers in a manufactory in China. Environ. Sci. Technol. 2015, 49 (11), 6953-62.

(6) Zhou, Z.; Shi, Y.; Vestergren, R.; Wang, T.; Liang, Y.; Cai, Y. Highly Elevated Serum Concentrations of Perfluoroalkyl Substances in Fishery Employees from Tangxun Lake, China. Environ. Sci. Technol. 2014, 48 (7), 3864-3874.

(7) Emmett, E. A.; Zhang, H.; Shofer, F. S.; Freeman, D.; Rodway, N. V.; Desai, C.; Shaw, L. M. Community exposure to perfluorooctanoate: relationships between serum levels and certain health parameters. J. Occup. Environ. Med. 2006, 48 (8), 771-9.

(8) Kishi, R.; Nakajima, T.; Goudarzi, H.; Kobayashi, S.; Sasaki, S.; Okada, E.; Miyashita, C.; Itoh, S.; Araki, A.; Ikeno, T.; Iwasaki, Y.; Nakazawa, H. The Association of Prenatal Exposure to Perfluorinated Chemicals with Maternal Essential and Long-Chain Polyunsaturated Fatty Acids during Pregnancy and the Birth Weight of Their Offspring: The Hokkaido Study. Environ. Health Perspect. 2015, 123 (10), 10381045 .

(9) Lau, C.; Anitole, K.; Hodes, C.; Lai, D.; Pfahles-Hutchens, A.; Seed, J. Perfluoroalkyl acids: A review of monitoring and toxicological findings. Toxicol. Sci. 2007, 99 (2), 366-394.

(10) SC-4/17: Listing of perfluorooctane sulfonic acid, its salts and perfluorooctane sulfonyl fluoride; http://chm.pops.int/ TheConvention/ThePOPs/ListingofPOPs/tabid/2509/Default.aspx.

(11) Chemicals proposed for listing under the Convention; http:// c h m.pops.in t/ The Convention/The P O P s / ChemicalsProposedforListing/tabid/2510/Default.aspx.

(12) Glynn, A.; Berger, U.; Bignert, A.; Ullah, S.; Aune, M.; Lignell, S.; Darnerud, P. O. Perfluorinated alkyl acids in blood serum from primiparous women in sweden: serial sampling during pregnancy and nursing, and temporal trends 1996-2010. Environ. Sci. Technol. 2012, 46 (16), 9071-9079.

(13) Olsen, G. W.; Lange, C. C.; Ellefson, M. E.; Mair, D. C.; Church, T. R.; Goldberg, C. L.; Herron, R. M.; Medhdizadehkashi, Z.; Nobiletti, J. B.; Rios, J. A.; Reagen, W. K.; Zobel, L. R. Temporal trends of perfluoroalkyl concentrations in American Red Cross adult blood donors, 2000-2010. Environ. Sci. Technol. 2012, 46 (11), 63308.

(14) Yeung, L. W.; Robinson, S. J.; Koschorreck, J.; Mabury, S. A. Part II. A temporal study of PFOS and its precursors in human plasma from two German cities in 1982-2009. Environ. Sci. Technol. 2013, 47 (8), 3875-82.

(15) Fu, J.; Gao, Y.; Cui, L.; Wang, T.; Liang, Y.; Qu, G.; Yuan, B.; Wang, Y.; Zhang, A.; Jiang, G. Occurrence, temporal trends, and halflives of perfluoroalkyl acids (PFAAs) in occupational workers in China. Sci. Rep. 2016, 6, 38039.

(16) Axmon, A.; Axelsson, J.; Jakobsson, K.; Lindh, C. H.; Jonsson, B. A. Time trends between 1987 and 2007 for perfluoroalkyl acids in plasma from Swedish women. Chemosphere 2014, 102, 61-7.
(17) Wang, M.; Park, J. S.; Petreas, M. Temporal changes in the levels of perfluorinated compounds in California women's serum over the past 50 years. Environ. Sci. Technol. 2011, 45 (17), 7510-6.

(18) Yeung, L. W.; Robinson, S. J.; Koschorreck, J.; Mabury, S. A. Part I. A temporal study of PFCAs and their precursors in human plasma from two German cities 1982-2009. Environ. Sci. Technol. 2013, 47 (8), 3865-74.

(19) Yeung, L. W. Y.; Mabury, S. A. Are humans exposed to increasing amounts of unidentified organofluorine? Environ. Chem. 2016, 13 (1), 102-110.

(20) Lu, Y.; Luo, B.; Li, J.; Dai, J. Perfluorooctanoic acid disrupts the blood-testis barrier and activates the TNF $\alpha / \mathrm{p} 38$ MAPK signaling pathway in vivo and in vitro. Arch. Toxicol. 2016, 90 (4), 971-983.

(21) Liu, H.; Sheng, N.; Zhang, W.; Dai, J. Toxic effects of perfluorononanoic acid on the development of Zebrafish (Danio rerio) embryos. J. Environ. Sci. 2015, 32, 26-34.

(22) Björkqvist, J.; Jämsä, A.; Renné, T. Plasma kallikrein: the bradykinin-producing enzyme. Thromb. Haemostasis 2013, 110 (3), 399-407.

(23) Feener, E. P.; Zhou, Q.; Fickweiler, W. Role of plasma kallikrein in diabetes and metabolism. Thromb. Haemostasis 2013, 110 (3), 434441.

(24) Long, Y.; Zhao, X.; Clermont, A. C.; Zhou, Q.; Liu, Q.; Feener, E. P.; Yan, B.; Jiang, G. Negatively charged silver nanoparticles cause retinal vascular permeability by activating plasma contact system and disrupting adherens junction. Nanotoxicology 2016, 10 (4), 501-511.

(25) Madsen, D. E.; Sidelmann, J. J.; Biltoft, D.; Gram, J.; Hansen, S. C1-inhibitor polymers activate the FXII-dependent kallikrein-kinin system: Implication for a role in hereditary angioedema. Biochim. Biophys. Acta, Gen. Subj. 2015, 1850 (6), 1336-1342.

(26) Clermont, A. C.; Chilcote, T. J.; Kita, T.; Liu, J.; Riva, P.; Sinha, S.; Feener, E. P. Plasma kallikrein mediates retinal vascular dysfunction and induces retinal thickening in diabetic rats. Diabetes 2011, 60 (5), 1590-1598.

(27) Simão, F.; Ustunkaya, T.; Clermont, A. C.; Feener, E. P. Plasma kallikrein mediates brain hemorrhage and edema caused by tissue plasminogen activator therapy in mice after stroke. Blood 2017, 129 (16), 2280-2290.

(28) Schmaier, A. H. The elusive physiologic role of factor XII. J. Clin. Invest. 2008, 118 (9), 3006-3009.

(29) Mitropoulos, K. A.; Esnouf, M. P. The autoactivation of factorXII in the presence of long-chain saturated fatty-acids - a comparison with the potency of sulfatides and dextran sulfate. Thromb. Haemost. 1991, 66 (4), 446-452.

(30) Li, H. J.; Leung, K. S.; Ballester, P. J.; Wong, M. H. istar: A Web Platform for Large-Scale Protein-Ligand Docking. PLoS One 2014, 9 (1), e85678.

(31) Trott, O.; Olson, A. J. Software news and update autodock vina: improving the speed and accuracy of docking with a new scoring function, efficient optimization, and multithreading. J. Comput. Chem. 2010, 31 (2), 455-461.

(32) Morris, G. M.; Huey, R.; Lindstrom, W.; Sanner, M. F.; Belew, R. K.; Goodsell, D. S.; Olson, A. J.; AutoDock4. and AutoDockTools4: Automated Docking with Selective Receptor Flexibility. J. Comput. Chem. 2009, 30 (16), 2785-2791.

(33) Colman, R. W.; Schmaier, A. H. Contact System: A Vascular Biology Modulator With Anticoagulant, Profibrinolytic, Antiadhesive, and Proinflammatory Attributes. Blood 1997, 90, 3819-3843.

(34) Ren, X. M.; Zhang, Y. F.; Guo, L. H.; Qin, Z. F.; Lv, Q. Y.; Zhang, L. Y. Structure-activity relations in binding of perfluoroalkyl compounds to human thyroid hormone T3 receptor. Arch. Toxicol. 2015, 89 (2), 233-42.

(35) Zhao, X.; Hao, F.; Lu, D.; Liu, W.; Zhou, Q.; Jiang, G. Influence of the Surface Functional Group Density on the Carbon-NanotubeInduced alpha-Chymotrypsin Structure and Activity Alterations. ACS Appl. Mater. Interfaces 2015, 7 (33), 18880-18890.

(36) Joensen, U. N.; Bossi, R.; Leffers, H.; Jensen, A. A.; Skakkebaek, N. E.; Jorgensen, N. Do perfluoroalkyl compounds impair human semen quality? Environ. Health Perspect. 2009, 117 (6), 923-927. 
(37) Zhang, W.; Lin, Z. K.; Hu, M. Y.; Wang, X. D.; Lian, Q. Q.; Lin, K. F.; Dong, Q. X.; Huang, C. J. Perfluorinated chemicals in blood of residents in Wenzhou, China. Ecotoxicol. Environ. Saf. 2011, 74 (6), 1787-1793.

(38) Olsen, G. W.; Burris, J. M.; Burlew, M. M.; Mandel, J. H. Plasma cholecystokinin and hepatic enzymes, cholesterol and lipoproteins in ammonium perfluorooctanoate production workers. Drug Chem. Toxicol. 2000, 23 (4), 603-620.

(39) Ng, C. A.; Hungerbuehler, K. Exploring the Use of Molecular Docking to Identify Bioaccumulative Perfluorinated Alkyl Acids (PFAAs). Environ. Sci. Technol. 2015, 49 (20), 12306-12314.

(40) Bischel, H. N.; MacManus-Spencer, L. A.; Zhang, C. J.; Luthy, R. G. Strong associations of short-chain perfluoroalkyl acids with serum albumin and investigation of binding mechanisms. Environ. Toxicol. Chem. 2011, 30 (11), 2423-2430.

(41) Hebert, P. C.; MacManus-Spencer, L. A. Development of a fluorescence model for the binding of medium- to long-chain perfluoroalkyl acids to human serum albumin through a mechanistic evaluation of spectroscopic evidence. Anal. Chem. 2010, 82 (15), 6463-6471.

(42) Zhang, L.; Ren, X.-M.; Guo, L.-H. Structure-Based Investigation on the Interaction of Perfluorinated Compounds with Human Liver Fatty Acid Binding Protein. Environ. Sci. Technol. 2013, 47 (19), 11293-11301.

(43) Kleszczyński, K.; Gardzielewski, P.; Mulkiewicz, E.; Stepnowski, P.; Składanowski, A. C. Analysis of structure-cytotoxicity in vitro relationship (SAR) for perfluorinated carboxylic acids. Toxicol. In Vitro 2007, 21 (6), 1206-1211.

(44) Hagenaars, A.; Vergauwen, L.; De Coen, W.; Knapen, D. Structure-activity relationship assessment of four perfluorinated chemicals using a prolonged zebrafish early life stage test. Chemosphere 2011, 82 (5), 764-772.

(45) Ren, X.-M.; Zhang, Y.-F.; Guo, L.-H.; Qin, Z.-F.; Lv, Q.-Y.; Zhang, L.-Y. Structure-activity relations in binding of perfluoroalkyl compounds to human thyroid hormone T3 receptor. Arch. Toxicol. 2015, 89 (2), 233-242.

(46) Weiss, J. M.; Andersson, P. L.; Lamoree, M. H.; Leonards, P. E. G.; van Leeuwen, S. P. J.; Hamers, T. Competitive Binding of Polyand Perfluorinated Compounds to the Thyroid Hormone Transport Protein Transthyretin. Toxicol. Sci. 2009, 109 (2), 206-216. 